This item was submitted to Loughborough's Research Repository by the author.

Items in Figshare are protected by copyright, with all rights reserved, unless otherwise indicated.

\title{
Fixed wing UAV survey coverage path planning in wind for improving existing ground control station software
}

PLEASE CITE THE PUBLISHED VERSION

https://doi.org/10.23919/ChiCC.2018.8482722

\section{PUBLISHER}

IEEE @ 2018 Technical Committee on Control Theory, Chinese Association of Automation.

\section{VERSION}

AM (Accepted Manuscript)

\section{LICENCE}

CC BY-NC-ND 4.0

\section{REPOSITORY RECORD}

Coombes, Matthew, Wen-Hua Chen, and Cunjia Liu. 2019. "Fixed Wing UAV Survey Coverage Path Planning in Wind for Improving Existing Ground Control Station Software". figshare. https://hdl.handle.net/2134/37151. 


\title{
Fixed Wing UAV Survey Coverage Path Planning in Wind for Improving Existing Ground Control Station Software
}

\author{
Matthew Coombes ${ }^{1}$, Wen-Hua Chen ${ }^{1}$, Cunjia Liu ${ }^{1}$ \\ 1. Department of Aeronautical and Automotive Engineering, Loughborough University, Loughborough LE11 3TU, U.K. \\ E-mail: M.J.Coombes@lboro.ac.uk,W.Chen@lboro.ac.uk,C.Liu5@lboro.ac.uk
}

\begin{abstract}
UAV technology is becoming increasingly mature and cheap. This recent cost reduction and performance improvement means that these systems have become reliable and cheap enough to be viable for mass use by farmers and operators for precision agriculture activities; such as disease identification, yield estimation and plant nitrogen monitoring.

A critical stage of this is the planning of the flight path which ensures complete image coverage of the region of interest. If the field is a simple convex shape, then generating an optimal coverage path is hugely simplified by using a back and forth Boustrophedon path. However, most fields have complex polygonal shapes, where planning the coverage path manually is nontrivial, as operators may not have the correct skills and experience. This is why in this paper, we outline an algorithm to improve the performance of survey path generation on complex ROI for mission planning software. The tools implemented in this paper take into account environmental factors and aircraft dynamics. By decomposing these complex survey regions into many smaller arrangements of manageable convex polygon survey regions, Boustrophedon paths can be used to cover them. By using a survey model for calculation of flight time in a wind field, it is used to optimise the decomposition in order to lower flight time.

The fastest survey path is used to generate waypoints files to be used with a number of popular mission planning software including: DJI PC Ground Control, Mission Planner, QGroundControl.
\end{abstract}

Key Words: Aerial Surveying, Remote Sensing, Polygon Decomposition, Coverage Path Planning, Boustrophedon paths, mission planning and ground control station.

\section{Introduction}

With advances in sensor and embedded hardware technology, we are seeing a huge proliferation in the use of UAVs across many applications. This includes the essential sector of agriculture. It has been shown that performing remote sensing from a UAV is a cheap and effective way of gathering GIS, and spectral image data of arable land. This data can then in turn be turned into actionable information, e.g. variable rate fertilizer of pesticide application. This could in turn lead to the efficiency gains needed to keep feeding the ever increasing population of Earth.

By affixing a small imaging system to either a small fixed or rotary wing platform, this enables low-cost remote sensing at low altitudes. They do not suffer from the same restrictive operational requirements of a manned aircraft, so can be flown much more frequently. A single image from low altitude will only cover a small area, but by taking a number of images across a Region of Interest (ROI), they can be stitched together using structure from motion photogrammetry software into a geo-referenced orthomosaic or Digital Elevation Model (DEM), which will give the desired GSD over the whole area [1].

The task of generating a path that a vehicle's coverage area will pass over all points of the ROI is called Coverage Path Planning (CPP). The simplest path that guarantees coverage of an area is a parallel simple back and forth motion of the vehicle along the long axis of the polygon. This is called a Boustrophedon path, which translated from greek means "way of the ox". This is a simple, effective path when performed within a simple convex polygonal area [2,3]. All that is required to construct this path is the ROI polygon, the sweep angle of the paths' cross track spacing determined by the UAVs image footprint, and required lateral overlap. Fig. 1 shows an example of a Boustrophedon path displaying the footprint of each photo and demonstrating that the

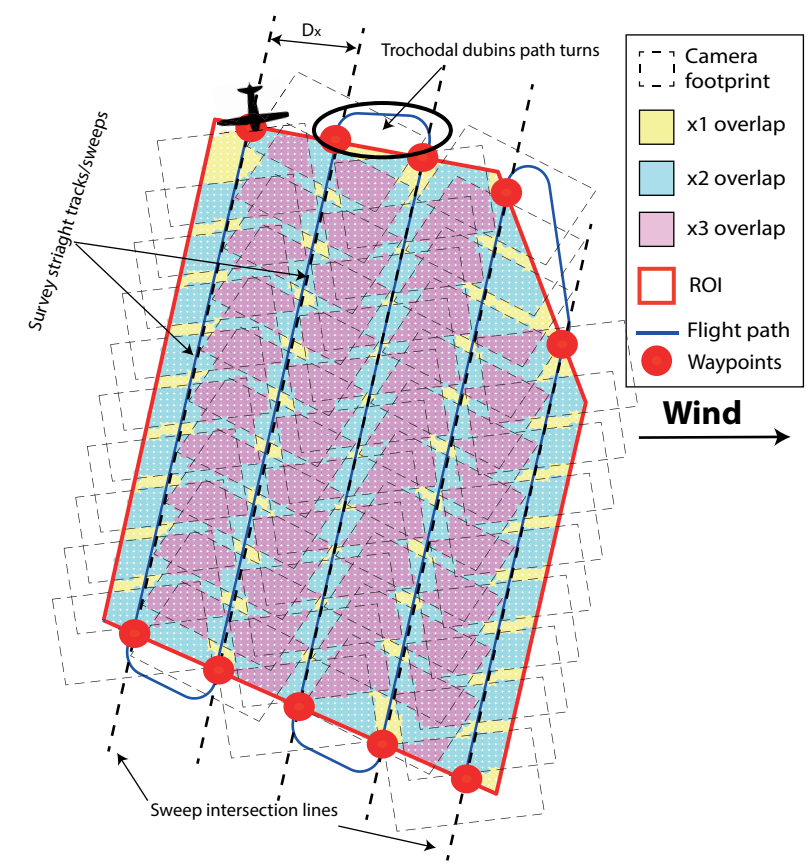

Fig. 1: Example Boustrophedon path of a convex polygon, showing the camera image footprints giving complete coverage. Sidelap $40 \%$, overlap $40 \%$. Notice the camera footprint angle relative to its track, this is due to the wind correction angle (crabbing) of the aircraft to stay on the track in the presence of wind

whole ROI is covered.

While mission planning tools exist to guide a fixed wing $\mathrm{UAV}$ on a path to image the entire Region of Interest (ROI), most are woefully inadequate for complex polygonal ROI shapes. It is reported in Finland that only 13\% of fields are convex [4], meaning that most arable farm fields will be too complex for standard mission planners. Most survey mission 
planners assume that the ROI is a simple polygon, and generate a Bousdphodon path for the convex hull of the ROI, example shown in Fig. 2a. This oversimplification leads to hugely increased flight times due to extended periods of flight outside the ROI. This will be discussed in greater detail in Section 2.

These planners are generally contained within Ground Control Station (GCS) software, e.g. Mission Planner ${ }^{1}$, QGround Control $^{2}$ and DJIFlightPlanner ${ }^{3}$. These all enable users to select the ROI which needs to be surveyed, and given user entered flight, camera, and survey parameters, will generate a set of sub-optimal waypoints that can be uploaded to the survey UAV.

If however, the ROI was decomposed into multiple smaller convex polygons to be surveyed sequentially, this would save huge amounts of time enabling operators and farmers to be much less experienced. There are a number of examples of polygon decomposition literature however they tend to be for terrestrial activities e.g. ground vehicles, lawn mowing and milling [5,6]. There has been very little literature for fixed-wing UAV surveys, one of the few works on a non Bousdphodon CPP meahod based on information gain. While this guaranteed coverage, it gave increased flight times. Also it requires a camera gimbal as the aircraft is in turns for a large portion of the flight [7]. An example of a simple concave ROI being decomposed into a smaller number of suitable convex polygons is shown in Fig. $2 b$.

This is achieved by first over-segmenting the polygon using trapezoidal decomposition [8], then recombining them in accordance with the Boustrophedon Cell Decomposition (BCD) detailed in [2]. As the trapezoidal decomposition is always performed vertically, there will be a unique decomposition for each rotation of the ROI polygon. This search space can be searched to find the rotation and decomposition which results in the lowest flight time.

The flight time for a given rotation can be calculated using previously developed techniques by this author. A flight time survey model in a steady uniform wind field was developed and validated in [9]. In simulation wind was shown to dramatically effect flight time, the simulation are presented in [9], and in experimentally in [3].

From the convex ROI output from BCD, the path and flight times can be calculated for each. Then each decomposed ROI be combined to give the path and the flight time for the whole original concave area.

In section 2 commercially available autopilots, and GCS are discussed, where their mission planning functions are discussed in comparison to the proposed algorithm. In section 3 CPP in wind is discussed. In Section 4 the extended BCD algorithm is laid out. In Section 5 a few example ROIs are decomposed and a CPP is generated. The survey flight times for the proposed algorithm are compared to the previous GCS convex hull technique. In Section 6 some concluding remarks are made.

\section{Ground control Stations \& Survey UAV systems}

There are a vast array of autopilot systems, some for hobbyists e.g. Pixhawk ${ }^{4}$, Papparazzi ${ }^{5}$, ArduPilotMega ${ }^{6}$ etc. For the commercial market, things are much the same e.g. MicroPilot $^{7}$, Vector from: UAVNavigration or Skyciruits etc.

We will be discussing the use and integration with the hobbyist autopilots, however CPP is just as useful to the commercial products. However as all their software is proprietary, integration with them there can be difficult, due to unknown path definitions and path following algorithms.

Ardupilot and PX4 autopilot software stacks (implemented on ArdupilotMega or Pixhawk) both use a nonlinear guidance logic for the path following [10]. A continuous path is defined by sending the autopilots discrete waypoints with latitude, longitude and altitude information. These waypoints can be generated and uploaded to the aircraft from GCS software mission planning tools. The waypoints are generated where the tracks intersect with the ROI [9], all the GCS software in question use this technique.

Seen in Fig. 3 how a ROI and the parameters of the aircraft, survey and camera are defined and entered into the mission planning tab of the GCS. From this it will calculate the camera footprint size and from that, define the distance between the survey tracks.

As mentioned previously, the Boustrophedon paths are optimal for convex polygons, however a concave polygon with no maximum vertex count can be defined in all these GCS. All GCSs that have been investigated simplify the concave polygon to its convex hull and plans a path at a survey angle (the rotational angle that all the parallel survey tracks are relative to north). A convex hull is the subset of points in Euclidean space that has the smallest set of points that create a convex polygon [11].

QGround Control and Mission planner are designed to be used with MavLink based rovers, multi/single rotary wing aircraft, fixed wing aircraft and even boats. Their mission planning functions require the user to define the survey angle, this adds another layer of expertise the user needs. UCGS does the same, however the user can select the survey angle or it can be snapped to the long axis of the polygon as described in [12]. This is shown to minimise the number of turns, where the minimum turns has been proven to minimise flight time in zero wind in the same paper. DJIMissionPlanner gives the user the ability to define the angle themselves or have the program do a survey angle optimisation sweep to find a more optimal mission. The user can select from three cost functions: minimum flight distance, minimum turns and minimum image count for total coverage.

Using the convex hull technique hugely simplifies the problem from one that is NP-Hard, to one that only has one solution for each survey angle, reducing the computational time. For simple concave polygons for most cases, the efficiency loss is minimal, but for complex concave polygons there can be huge flight time savings, discussed further in Section 5 .

\footnotetext{
${ }^{4} \mathrm{http}: / / \mathrm{px} 4 . \mathrm{io} /$

${ }^{5}$ http://paparazzi.enac.fr/wiki

${ }^{6}$ http://ardupilot.org/

${ }^{7}$ https://www.micropilot.com/

\footnotetext{
${ }^{1}$ http://ardupilot.org/planner/

${ }^{2} \mathrm{http}: / /$ qgroundcontrol.com/

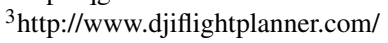




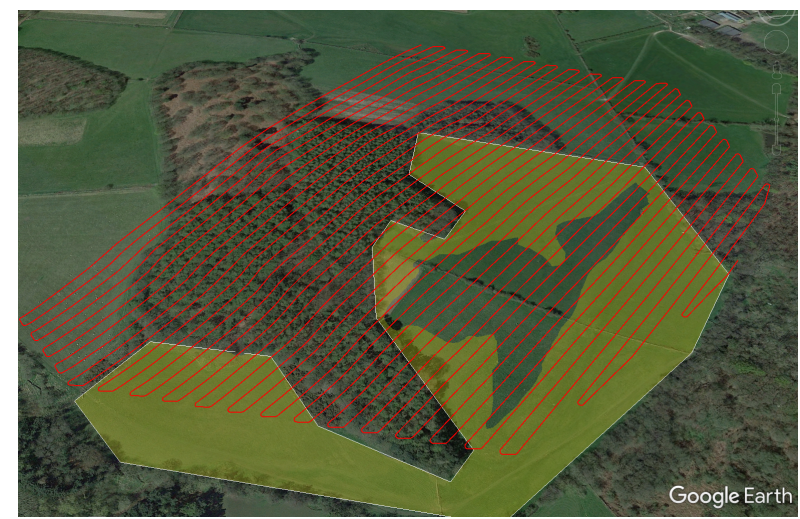

(a) GCS convex hull CPP technique

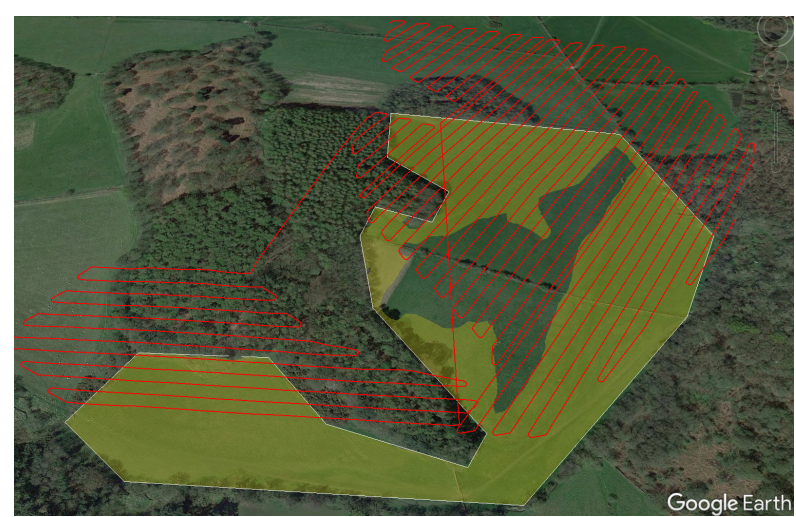

(b) Proposed technique

Fig. 2: Comparison of example survey mission plan from GCS vs the BCD meahod

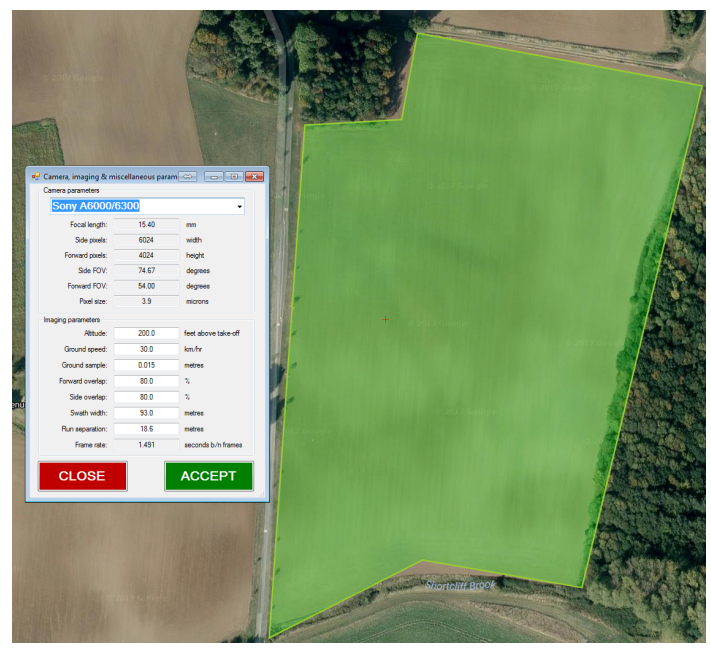

Fig. 3: DJIMissionPlanner Survey planner interface, selection of ROI, and user entry of camera, survey and aircraft parameters

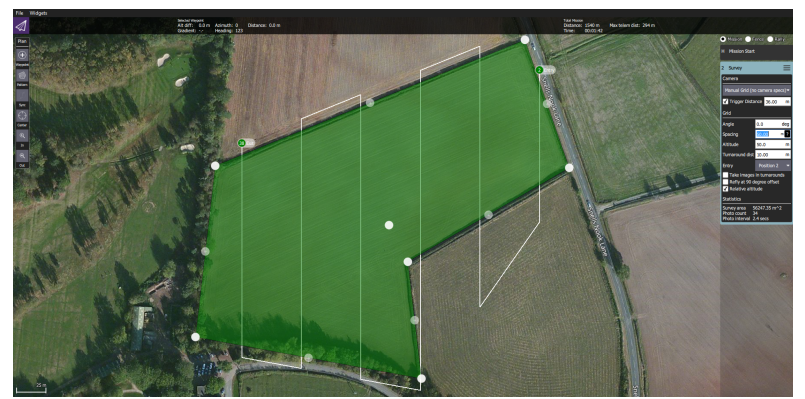

Fig. 4: QGround Control generates waypoints for the ROI (green) where the parallel track intersect with the edge of the ROIs convex hull

\section{Coverage Path Planning}

The most efficient path to cover a convex polygon is a Boustrophedon path. These consist of back and forth motions at a particular survey angle across a ROI. This back and forth path ensures total photo coverage. The path consists of two different states of flight; the straight sweep paths (when the photos are taken), and the turn manoeuvre used to transition to the next sweep. This is laid out in detail in [9], and such will only have a brief overview here.

The distance between tracks $D_{x}$ needs to be found (also shown in Fig. 1). If the user has a required Ground Sample Distance (GSD), then $D_{x}$ can be calculated below:

$$
D_{x}=2 \tan \left(\frac{f_{o v_{x}}}{2}\right) \frac{\left(1-w_{s}\right) N_{x} G S D}{f o v_{x}}
$$

where $h$ is the height of the aircraft above the ground, GSD is in $\frac{m}{p i x}, f o v_{x}$ is the horizontal angular Field Of View (FOV) of the sensor, $N_{x}$ is the number of pixels the sensor has in the horizontal direction.

The survey sweep angle $\left(\psi_{s}\right)$ is the direction of the parallel straight tracks. The angle is important in order to minimise the number of turns needed. So to lower the search space $\psi_{s}$ is fixed to the angle of the long axis of the polygon. This angle is calculated using a minimum area bounding box, calculating its long axis angle as described in [9].

Sweep track lines are placed evenly across the ROI at intervals of $D_{x}$, rotated by $\psi_{s}$, shown in Fig. 1. Where the lines intercept with the ROI polygon, a waypoint is placed, these waypoints define the start and end of the survey straight line tracks. At the end of each track, the aircraft have to perform a $180^{\circ}$ turn manoeuvre to get on the next survey track.

In order to calculate the total flight time in this convex survey, the geometry of the survey and the speed of the aircraft must be known at all stages. As if path length and ground speed is known, then flight time in each stage can be found. This is fully detailed in [9] but has a brief overview in the rest of this section.

\subsection{CPP in wind}

Wind can have a significant effect on small aircraft; the windspeed experienced by a small UAV can easily be $50 \%$ of the airspeed. As a result, it is vital to account for wind for small survey UAVs as huge time flight time savings can be gained

By assuming that the wind field is steady and uniform, accounting for the wind is simply a matter of converting from the wind frame to the ground frame. Using the wind triangle, an equation for aircraft's ground speed along the sweep portion of the survey: 


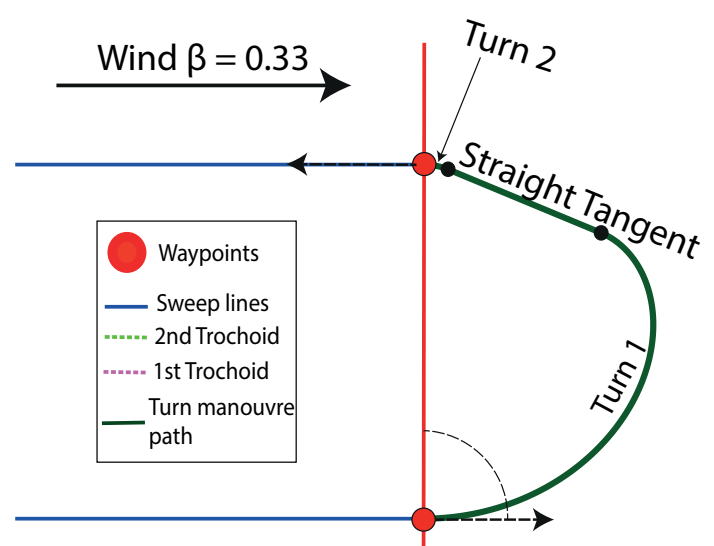

Fig. 5: Trochoidal turn manoeuvre to transit between one straight line survey track to the next.

$$
\left.V_{g}=V \cos \left(\arcsin \left(\left(\frac{V_{w}}{V}\right) \sin \left(\psi_{w t a}\right)\right)\right)\right)+\left(V_{w} \cos \left(\psi_{w t a}\right)\right.
$$

where $\mathrm{V}$ is the aircraft's airspeed, and suffix $w$ is wind speed, $g$ ground speed and $\psi_{w t a}$ is the aircraft's relative wind angle where $\psi_{w t a}=\psi_{g}-\psi_{w} . \psi_{g}$ is the ground track angle of the aircraft.

\subsection{Trochoidal Turn Paths}

Detailed in [13] is a meahod to fly a minimum time turning path in wind. It extends the Dubins path's circular turns with trochoidal shaped turns. Dubins paths consist of an initial turn, a final turn and a straight tangential line between them, seen in Fig. 5. The equations to generate the trochoidal version of this are based on finding the angle of tangency between the trochoids of the initial turn and final turn. Details on the specifics is laid out in [9].

The total time for the whole manoeuvre is the summation of the time in both turns and in the straight tangent. Where the time in the straight can be calculated for the length of the line, and the ground speed of the aircraft as found from Eq. (2). Where the time is calculated from the angle subtended over the trochoid and its known constant turn rate.

If the time for all straight tracks, and turn manoeuvres are summed together, the total time of the individual convex survey in wind can be calculated.

\section{Polygon Decomposition}

In order to minimise the flight time relative to the previous convex hull technique, only the area of the ROI polygon needs to be surveyed. This means decomposing the concave polygon into smaller convex ones that will be surveyed using a Boustrophedon path. This is initially done using trapezoidal decomposition. This over segments the polygon, many of the new cells are to be recombined to give convex and more efficiently surveyable polygons. This is done using spilt and merge points in the decomposed cells adjacency graph. When a final decomposition has been obtained, the long axis angle for each cell is calculated and using the survey model from Section 3, the path and flight time are calculated. This is repeated for each desired polygon rotation angle. The polygon rotation with minimum flight time is the chosen decomposition and the final CPP can be generated.
Using a very complex concave ROI with 21 vertices, 9 of which are concave is used here to demonstrate the full BCD decomposition. Seen in Fig. 6 is the initial trapezoidal decomposition segmenting it into 19 separate convex cells. The undirected adjacency graph representing the connectivity of each cell is also shown. The graph is used in the recombination phase.

There are a number of techniques that could be used in the over-segmentation stage (triangular, visibility based, approximate decomposition), however the long narrow shape of trapezoids lend themselves very well to efficient survey paths. This is because the long narrow shape means less wasteful turns are needed [12]. This plane sweep based trapezoidal decomposition algorithm works by generating a vertical sweep line at each vertex in the direction of increasing $x$. New vertices are created where the sweep line intersects with the polygon edges, which creates the new convex polygons. An overview of this algorithm is detailed in [8].

Using a modified version of the BCD technique from [2] many of the cells produced can be recombined. It is based on splits and merges in the cell adjacency graph shown on the right side of Fig. 6. For example, cells $\{1,6,7\}$, if surveyed individually this would be inefficient, but if combined they give a single larger convex polygon, which is a much faster survey. These subgraphs can be determined by splits and merges in the graph. It can be seen that there is a split in the graph after node/cell 7 . This means that the subgraph $\{1,6,7\}$ can be merged together to make a single polygon. This polygon is shown in on the left image in Fig. 7 as cell 1. Continuing on to the next example subgraph of $\{8,12\}$, this is after a split and just before another split in the graph, so these are merged into cell 4 in Fig. 7.

In order to expand the search space, the ROI polygon is rotated initially, then decomposed, then rotated back to its real world orientation after the decomposition is complete. Note that the wind angle also needs to be rotated before the survey model is applied to the decomposition. This is so its relative angle to the polygon does not change. Each vertex of the polygon must be rotated about polygon rotation angle $\psi_{p}$. Let $P$ be a polygon with $n_{v}$ verities $\nu=\left\{v_{1}, v_{2} \ldots v_{n_{v}}\right\}$, then $P$ can be rotated as shown in Eq. (3), where $P_{r}$ is the rotated Polygon, $P$, and $\psi_{p}$ is the angle of rotation.

$$
P_{r}=\left[\begin{array}{cc}
\cos \psi_{p} & -\sin \psi_{p} \\
\sin \psi_{p} & \cos \psi_{p}
\end{array}\right] P
$$

For each polygon rotation, the flight time for its BCD is calculated, and the rotation with the minimum value is the final decomposition and coverage path, as per Eq. (4).

$$
T\left(\psi_{p}\right)=\min \left\{\sum_{i=1}^{n} F T I W\left(\psi_{p}\right)\right\}
$$

where $\mathrm{T}$ is total flight time, $i$ is the current cell index, $n$ is the total number of cell after the initial decomposition and FTIW is the flight time in wind function defined in Section. 3.

\section{Simulation results}

A direct comparison between the previous technique and the proposed one is performed in this section. This is all 


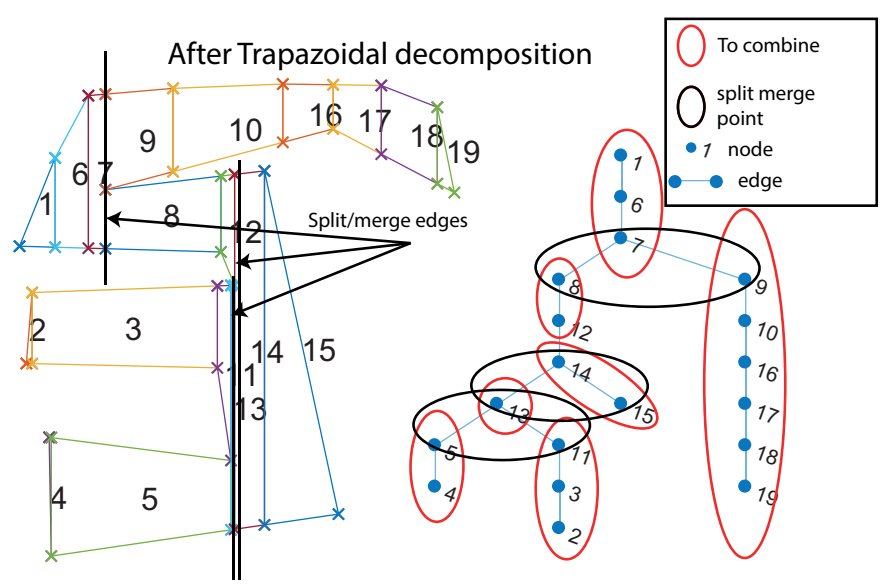

Fig. 6: Left: Trapezoidal decomposition of complex concave polygon, Right: cell adjacency graph. Merge/Split points for the recombination phase is also shown

polygon decoposed cells and paths decoposed cell adjecency graph
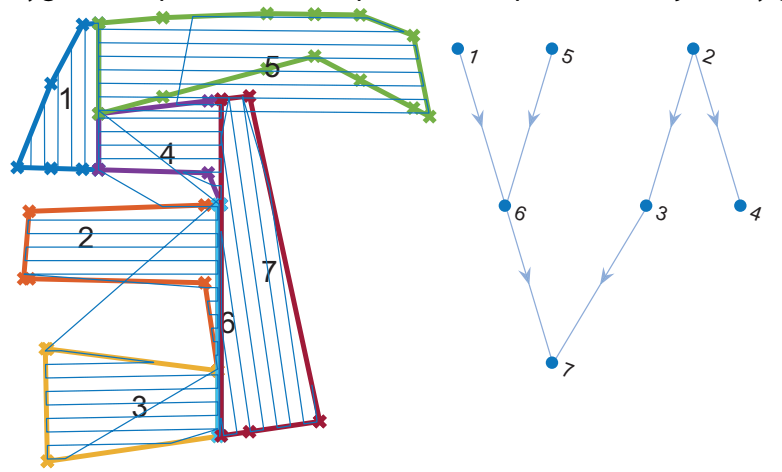

Fig. 7: Same survey simulation as Fig. 6, but a stronger 4 $\mathrm{m} / \mathrm{s}$ westerly wind is used

performed with the survey model from Section 3, these numerical simulations are computed in MatLab. The survey, camera and aircraft parameters are as follows:

$$
\begin{array}{llll}
\psi_{w}=180^{\circ} & V_{w}=2 \mathrm{~m} / \mathrm{s} & V=8 \mathrm{~m} / \mathrm{s} & \dot{\psi}=0.7 \mathrm{rad} / \mathrm{s} \\
D_{x}=33.7 \mathrm{~m} & w_{s}=0.6 & h=60 \mathrm{~m} & G S D=0.0123
\end{array}
$$

As an example, the ROI from Fig. 4 as an example field to survey. The BCD decomposition of this polygon is shown alongside the convex hull meahod in Fig. 8. From the close by launch point, the flight path for the best polygon rotation was generated and the flight time calculated at $289.8 \mathrm{~s}$, as opposed to the significantly longer time of $390 \mathrm{~s}$ for the convex hull technique. It is obvious that the reduction in total coverage area is responsible for the reduction in flight time. Any fixed wing aircraft would have no issue flying for an extra 100s when the flight is only 7 mins. However when the flight is much longer, the $25 \%$ flight time saving become much more significant.

Furthermore if the ROI is much more complex, then the proposed algorithm is essential as the flight would be prohibitively long using the convex hull technique. For example the polygon in Fig. 9 which has 10 concave vertices. During these long flights the aircraft is exposed to the wind for longer, so its influence will be much greater. To show this different wind conditions are also tested.
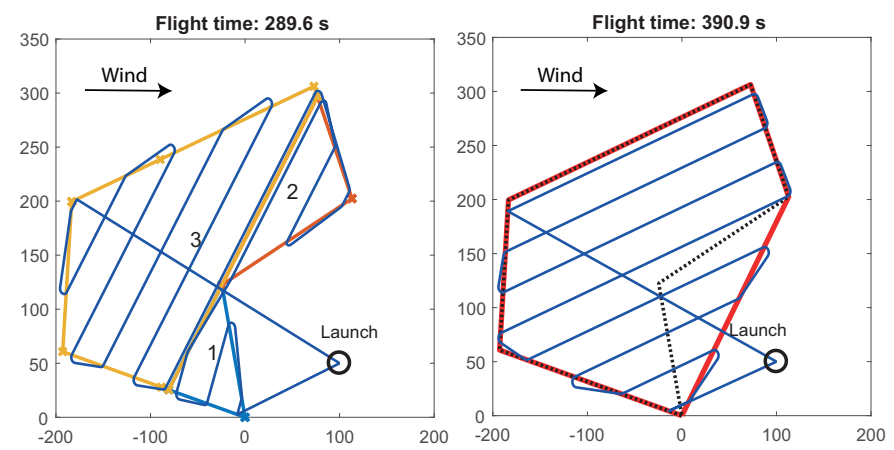

Fig. 8: Left: BCD, Right: Convex Hull. Westerly wind of 3 $\mathrm{m} / \mathrm{s}$
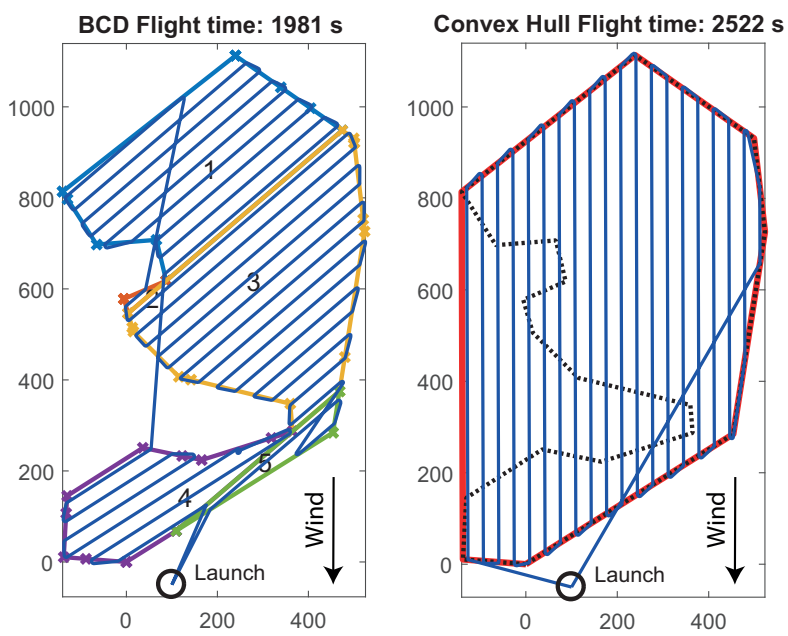

Fig. 9: Complex ROI BCD Decomposition, and Convex Hull path planning. All in a northerly wind of $2 \mathrm{~m} / \mathrm{s}$

For the more complex polygon in Fig. 9 the flight time is reduced from 2522s for the previous convex hull technique to 1980 s for BCD which is over 8 mins, or $19 \%$ time saving. This simulation was rerun with double the windspeed at $4 \mathrm{~m} / \mathrm{s}$ then rotated to be from the south. The stronger wind raised the single convex hull solution flight time to $2817 \mathrm{~s}$ $\frac{m}{p i x}$ shown in Fig. 10. The flight time increased to 2295 s for the BCD solution, also the best rotation angle is $345^{\circ}$. As detailed in [9], sweep angles perpendicular to the wind have a flight time advantage over surveying parallel to the wind direction. This is why the algorithm tends to find lower flight times for polygon rotations close to perpendicular to the wind, which is seen in both examples. A summary of all these results are in Table 1.

In order to re-integrate this with the GCS, discrete waypoints need to be generated. The position of the start and end point of each straight line track can be used with a reference latitude and longitude to define all the waypoints needed. This is shown in Fig. 11.

\section{Conclusion}

All commercially available GCS software's mission planning functions are inadequate to plan CPP missions for complex polygonal ROIs. The convex hull simplification used increases the flight time to acceptable levels in these cases. This paper proposes a technique, to decompose the ROI into a smaller more efficient shape for CPP. By using a previously 
Table 1: Simulation Results

\begin{tabular}{|l|c|c|c|}
\hline Simulation description & Best Rotation & Flight Time BCD & Time Convex Hull \\
\hline Simple Concave, Wind:2m/s $180^{\circ}$ & $20^{\circ}$ & $289.8 \mathrm{~s}$ & $390.6 \mathrm{~s}$ \\
Complex Concave, Wind:2m/s $270^{\circ}$ & $40^{\circ}$ & $122 \mathrm{~s}$ & $2817 \mathrm{~s}$ \\
Complex Concave, Wind:4m/s $180^{\circ}$ & $345^{\circ}$ & $2100 \mathrm{~s}$ & $2590 \mathrm{~s}$ \\
\hline
\end{tabular}
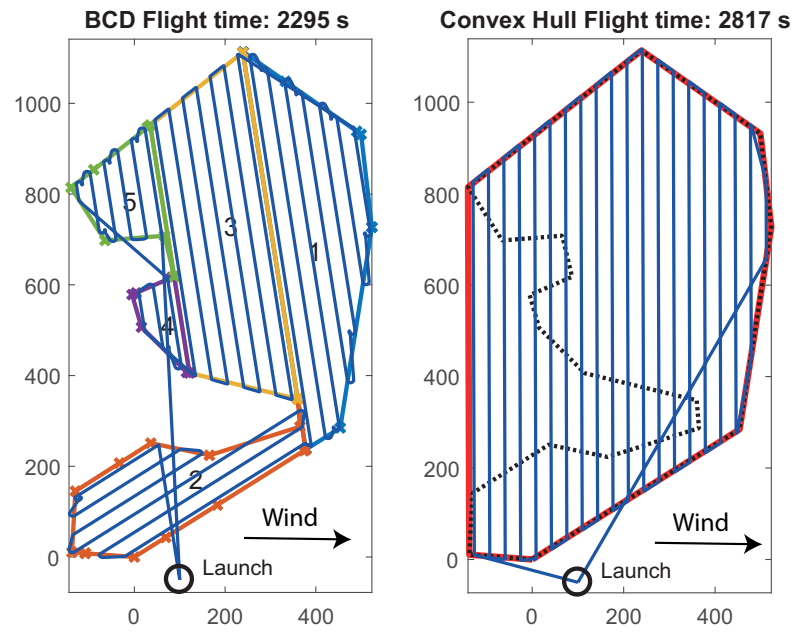

Fig. 10: Complex ROI BCD Decomposition, and Convex Hull path planning. All in a westerly wind of $4 \mathrm{~m} / \mathrm{s}$

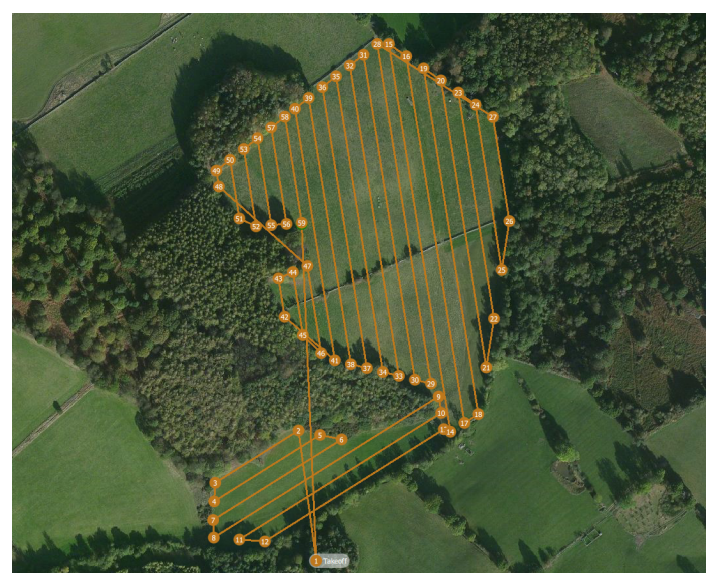

Fig. 11: CPP generated from proposed algorithm converted into discrete waypoints to upload to the UAV from QGround Control

developed survey in wind model, a minimum flight time optimisation can be preformed by decomposing the polygon at different rotations. Wind is shown to be very important to take account of.

The presented technique does not however guarantee $100 \%$ convexity, clearly seen in decomposed cell 5 in Fig. 7 , but it does guarantee a reduction of the number of concave vertices by at least the number of merge/split points. It is guaranteed to have a smaller coverage area than the convex hull technique.

\section{Acknowledgement}

This work was supported by Science and Technology Facilities Council (STFC) under Newton Fund with grant number ST/N006852/1.

\section{References}

[1] R Gini, D Pagliari, D Passoni, L Pinto, G Sona, and P Dosso. Uav photogrammetry: Block triangulation comparisons. Int. Arch. Photogram. Remote Sens. Spat. Inf. Sci, XL-1/W2:pp.157-162, 2013.

[2] Howie Choset. Coverage of known spaces: The boustrophedon cellular decomposition. Autonomous Robots, 9(3):247$253,2000$.

[3] A. Xu, C. Viriyasuthee, and I. Rekleitis. Optimal complete terrain coverage using an unmanned aerial vehicle. In 2011 IEEE International Conference on Robotics and Automation, pages 2513-2519, May 2011.

[4] Timo Oksanen and Arto Visala. Coverage path planning algorithms for agricultural field machines. Journal of Field Robotics, 26(8):651-668, 2009.

[5] Esther M Arkina, Sándor P Fekete, and Joseph SB Mitchella. Approximation algorithms for lawn mowing and milling. Computational Geometry, 17:25-50, 2000.

[6] R. N. De Carvalho, H. A. Vidal, P. Vieira, and M. I. Ribeiro. Complete coverage path planning and guidance for cleaning robots. In Industrial Electronics, 1997. ISIE '97., Proceedings of the IEEE International Symposium on, volume 2, pages 677-682 vol.2, Jul 1997.

[7] L. Paull, C. Thibault, A. Nagaty, M. Seto, and H. Li. Sensor-driven area coverage for an autonomous fixed-wing unmanned aerial vehicle. IEEE Transactions on Cybernetics, 44(9):1605-1618, Sept 2014.

[8] Jean-Claude Latombe. Robot motion planning, volume 124 Springer Science \& Business Media, 2012.

[9] M. Coombes, W. H. Chen, and C. Liu. Boustrophedon coverage path planning for uav aerial surveys in wind. In 2017 International Conference on Unmanned Aircraft Systems (ICUAS), pages 1563-1571, June 2017.

[10] Sanghyuk Park, John Deyst, and Jonathan P How. A new nonlinear guidance logic for trajectory tracking. In AIAA guidance, navigation, and control conference and exhibit, pages 16-19, 2004.

[11] Selim G. Akl and Godfried T. Toussaint. A fast convex hull algorithm. Information Processing Letters, 7(5):219 - 222, 1978.

[12] Yan Li, Hai Chen, Meng Joo Er, and Xinmin Wang. Coverage path planning for $\{$ UAVs $\}$ based on enhanced exact cellular decomposition method. Mechatronics, 21(5):876 885, 2011. Special Issue on Development of Autonomous Unmanned Aerial Vehicles.

[13] Laszlo Techy and Craig A Woolsey. Minimum-time path planning for unmanned aerial vehicles in steady uniform winds. Journal of guidance, control, and dynamics, 32(6):1736-1746, 2009. 